\title{
Specificity and sensibility of 9-Itens Wearing- off Questionnaire in Brazilian Parkinson disease patient sample
}

\author{
Especificidade e sensibilidade do Questionário Wearing-off 9-Itens em amostra brasileira \\ de pacientes com doença de Parkinson
}

Jasper Guimarães Santos, Hsin Fen Chien, Egberto Reis Barbosa

\begin{abstract}
Objective: (1) To evaluate whether the Nine Items Questionnaire (WOQ-9) for the detection of wearing-off (WO) in Parkinson Disease (PD), by means of its screening ability, is a helpful tool to assist neurologists in diagnosing WO; (2) To determine the sensitivity and the specificity of a free Brazilian Portuguese translation of WOQ-9. Method: A sample obtained by convenience included 60 patients. The WOQ-9 was answered by the patients themselves before their routine consultations. The detection of the WO by the WOQ-9 was compared with the neurologist assessment. Statistical significance was 5\%. Results: The WOQ-9 showed sensitivity of 100\%, specificity of 10.3\%, positive and negative predictive values of $54.4 \%$ and $100 \%$ respectively. The identification of WO by the WOQ-9 was congruent in $54.5 \%$ of cases with neurological evaluation. Conclusion: The WOQ-9 is a convenient screening tool to aid physicians to detect WO in PD patients, and it is a quick and easy self-administered questionnaire.
\end{abstract}

Keywords: Parkinson's disease, levodopa, wearing-off, questionnaire.

\section{RESUMO}

Objetivo: (1) Verificar se o Questionário de Nove Itens (WOQ-9) para detectar wearing-off (WO) na doença de Parkinson (DP), pela sua capacidade de triagem, seria útil aos neurologistas na identificação de WO; (2) determinar a sensibilidade e especificidade da versão livre em Português Brasileiro do WOQ-9. Método: Ao todo 60 indivíduos com DP compuseram uma amostra obtida por conveniência. Os próprios pacientes responderam ao WOQ-9 antes de suas consultas rotineiras. A detecção de WO pelo WOQ-9 foi comparada com a avaliação neurológica. A significância estatística foi 5\%. Resultados: O WOQ-9 apresentou sensibilidade de 100\%, especificidade de 10,3\%, valores preditivos positivo e negativo de 54,4\% e 100\% respectivamente. A identificação de WO pelo WOQ-9 foi congruente em 54,5\% dos casos com a avaliação neurológica. Conclusão: O WOQ-9 é um método de rastreamento útil para identificar WO em pacientes com DP, e é um questionário de autoavaliação cuja aplicação é fácil e rápida.

Palavras-chave: doença de Parkinson, levodopa, wearing-off, questionários.

The treatment of Parkinson's disease (PD) remains a major challenge spite of the increasing therapeutic arsenal in recent decades and progressing knowledge of its pathophysiology. Levodopa (LD) remains the most effective and widely prescribed drug in the symptomatic treatment of $\mathrm{PD}^{1}$. Currently it is acknowledged that up to $50 \%$ of patients have motor fluctuations after two years of the introduction of $\mathrm{LD}$ or another dopaminergic agonist (DA $)^{2,3}$.

The long-term use of levodopa leads to complications directly related to the duration of the treatment. Such complications include motor symptoms (MS) and non-motor symptoms (NMS). The most frequent motor complications are the motor fluctuations and dyskinesias. Wearing-off (WO) is one subtype of motor fluctuation and occurs over time, when the effectiveness of a dose of dopaminergic agonist tends to wear off earlier than before. For confirmation of this phenomenon, WO symptoms should improve after the intake of the next dose of antiparkinsonian drug $(\mathrm{AD})^{2}$.

The physiopathology of WO is controversial; it could be due to the decrease of the pre-synaptic dopamine receptors with consequent diminished storage capacity of dopamine in the striatum, or beyond post-synaptic mechanisms as the interruption of signal transduction for synthesis of proteins mediated by D1 and D2 receptors, or because of

Departamento de Neurologia, Faculdade de Medicina, Universidade de São Paulo, Sao Paulo SP, Brazil.

Correspondence: Jasper Guimarães Santos; Rua Alves Guimarães, 643 / ap. 31; 05410-001 São Paulo SP, Brasil; E-mail: santosjasper@gmail.com Conflict of interest: There is no conflict of interest to declare.

Received 15 April 2014; Received in final form 24 July 2014; Accepted 12 August 2014. 
dopamine depletion due to neuronal loss. However, the loss of the physiological integrity of the outflow tracts of the basal ganglia is the fundamental prerequisite for the emergence of WO. The reduction and later extinction of the phasic release of dopamine by the nigrostriatal system, culminates with a tonic stimulation of postsynaptic dopamine receptors ${ }^{4}$. After the introduction of $\mathrm{LD}$ therapy, the improvement of PD symptoms follows two patterns undistinguishable clinically in the initial stages of the treatment. Firstly the improvement of the symptoms begins rapidly, lasts for an average of 4 to 5 hours after ingestion of the medication and provides relief mainly of the MS, this is known as "short duration pattern". Secondly, occurs the "long duration pattern", when a prolonged improvement of the symptoms after dopaminergic stimulation is observed, generally obfuscated in the initial stages of the treatment with LD or DA, and this effect may sustain up to fourteen days after stopping the $\mathrm{AD}^{5}$.

The early recognition of WO allows the treatment of this complication in less advanced stages of PD, reduces the emergence of complications related to WO and may delay its progression ${ }^{6}$. Strategies to ameliorate WO includes the optimization of dopaminergic therapy and/or the early introduction of catechol-O-methyl transferase (COMT) inhibitors in association to LD. Studies showed that either treatment option have similar results to improve motor fluctuation ${ }^{7,8,9,10}$.

Many studies have verified whether questionnaires answered by patients are helpful to enhance the detection of $\mathrm{WO}^{11,12,13,14}$. This study aimed to assess whether WOQ-9 is an useful tool to assist neurologists to detect WO in clinical practice, increasing its identification. The sensitivity and specificity of the WOQ-9 to detect WO were determined by comparing the identification of WO by the clinical evaluation performed by neurologists. We also analyzed which symptoms of the WOQ-9 were more frequent and disabling, and the prevalence of other symptoms not included in the WOQ-9.

\section{METHOD}

This was a cross-sectional study conducted at the Movement Disorders Clinic of the Department of Neurology of the University of Sao Paulo School of Medicine during the period of May 2011 to May 2013, and it was approved by the local ethical committee.

The WOQ-9 was translated from English into Brazilian Portuguese but not formally validated. WO was present if the patient checked at least one symptom of the Nine Items Questionnaire for WO (WOQ-9). Moreover, it was confirmed if the patient's chosen symptoms improved after the intake of the next $A D^{13}$. The clinical assessment was the gold standard method for diagnosing WO. Sensitivity was defined as the probability that a PD patient, presenting WO according to clinical assessment, be sorted as having WO by WOQ9 and specificity was defined as the probability that a PD patient without WO ascertain by physician would be classified as being without the condition by the WOQ-9. The positive predictive value (PPV) and negative predictive value (NPV) represented the likelihood of subjects with WO assessed by WOQ-9 truly experienced WO or not, respectively, when compared with the clinical assessment.

\section{Study population}

A non-probabilistic sample was obtained by convenience. All individuals had idiopathic PD (according to the UK Parkinson's Disease Society Brain Bank Clinical Diagnostic Criteria ${ }^{15}$ ) and fulfilled the following inclusion criteria: at least 30 years old, literate, duration of disease of at least 2 years, use of LD and/or DA, Hoehn and Yahr scale score (HY) $<5^{16}$, and to have provided written informed consent. Patients with cognitive impairment or severe psychiatric co-morbidity were excluded.

\section{Study design}

Seventy-five patients were invited to participate to the study. However, while answering the questionnaire, eleven were excluded due to functional illiteracy and four because they presented cognitive impairment or severe psychiatric co-morbidity. At the conclusion of the study the final sample was composed of 60 individuals.

The patients answered to the WOQ-9 on the same day, before their routine neurological consultation. One specialist in movement disorders, named investigator, instructed the patients to mark an " $x$ " to "yes" if they had any of the nine symptoms and "no if none of them was present. The investigator explained that if the answer was "yes" the patient had to check an "x" to "yes" or "no" if symptoms improve or not after taking the next $\mathrm{AD}$, respectively. If the answer was "no" patients would not need to proceed to the second question about the corresponding symptom. These instructions were given to all patients by the investigator who had his function limited to supervise the patient's task of filling out the questionnaire. The educational level of the patients was ascertained and scored according to the number of years studied (studied years).

After completion of WOQ-9, each individual would also read and answer two questions: - Which are the worst symptoms in the questionnaire for you? and - Do you have any other disabling symptom which is not listed on the questionnaire?

The standardized clinical assessment was carried out by neurologists who were blinded to the study objectives. The investigator, soon after consultations, subtly inquired to neurologists: - "The patient who you just saw shows wearing off?" The results obtained through WOQ-9 were compared with the responses of the physicians. 
The Unified Parkinson's Disease Rating Scale (UPDRS) ${ }^{17}$ part I to IV was applied to all patients in "on" state. The investigator inquired how long has he/she taken the $\mathrm{AD}$ and if it was near the time for the next dose. The "on" state was attributed to the patient when he/she was in their best condition with the effect of the medication. Their stages at Hoehn and Yahr modified scale (HY) were also obtained. The Sum variable was performed by the sum of symptoms that improved after the next medication dose, and used in the correlation analysis.

\section{Data analysis}

The descriptive analysis of all variables of the study was performed. The qualitative analyses were expressed by absolute and relative frequency. Quantitative variables were expressed in terms of its values of central tendency and dispersion. In order to verify the homogeneity of variances and the adherence to the normal curve Levene test and Kolmogorov-Smirnov test were used respectively. The Spearman correlation coefficient was used to assess whether there was correlation between the presence of WO according to WOQ-9 and age, educational level, disease duration, total UPDRS and Hoehn and Yahr stage. In order to compare the questionnaire to the gold standard method we calculated the sensitivity, specificity, positive and negative predictive values. To verify the association between the qualitative variables, such as the presence of a symptom detected by the physicians and the WOQ-9, we used Fisher's exact test. The significance level was $5 \%^{18}$. The statistical software utilized was: SPSS 17.0 for Windows.

\section{RESULTS}

The WOQ-9 was answered by sixty patients and their age ranged from 31 to 89 years old (mean=60.1, standard deviation=13.9). Thirty five patients were male $(58.3 \%)$ and twenty-five were female (41.7\%). The duration of the disease varied from 3 to 16 years (mean=9.9, standard deviation $=5.5$ ). The educational level ranged from 2 to 19 studied years (mean $=8$, standard deviation $=4.45$ ).

Homogeneity between the answer given by the physician and the detection of WO by WOQ-9 was observed (Fisher's exact test, $\mathrm{p}<0.05$ ). The congruence for the detection of WO by the physicians and WOQ-9 was obtained in $54.5 \%$ of the cases. In $45.6 \%$ of the cases WO was identified only by the WOQ-9, as shown in the Table 1.

With regard to the questionnaire, it showed a sensitivity of $100 \%$, specificity of $10.3 \%$, PPV of $54.4 \%$, and NPV of $100 \%$ (confidence interval of 95\%). Receiver Operator Curve (ROC) curves were plotted to analyze the results of sensitivity and specificity. The curves demonstrated statistical significance
Table 1. Association analysis between qualitative variables by Fisher's Exact Test.

\begin{tabular}{|c|c|c|c|}
\hline \multirow{2}{*}{$\begin{array}{l}\text { Physician } \\
\text { evaluation }\end{array}$} & \multicolumn{2}{|c|}{ WOQ-9 } & \multirow{2}{*}{ Total } \\
\hline & Yes & No & \\
\hline \multicolumn{4}{|l|}{ Yes } \\
\hline$N^{\circ}$ & 31 & - & 31 \\
\hline$\%$ & 54.4 & - & 54.0 \\
\hline \multicolumn{4}{|l|}{ No } \\
\hline$N^{\circ}$ & 26 & 3 & 29 \\
\hline$\%$ & 45.6 & 100.0 & 48.3 \\
\hline \multicolumn{4}{|l|}{ Total } \\
\hline$N^{\circ}$ & 57 & 3 & 60 \\
\hline$\%$ & 100.0 & 100.0 & 100.0 \\
\hline
\end{tabular}

for the detection of WO by WOQ-9 when analyzed individually, but not for the detection of WO by neurological evaluation (Figure 1).

The most and least frequent symptoms were Any Slowness in Movement (93.3\%) and Pain/Aching (65.0\%), respectively. The frequency of each WOQ-9 symptom is shown in Figure 2.

The symptoms mostly appointed as "the worst" by the patients were: Tremor (25.6\%) and Any Stiffness (24.4\%), as shown in Figure 3. When questioned if there were any other disabling symptoms but was not listed on the WOQ-9, the patients diverged considerably but they reported mostly Gait Disorders (7.7\%) and Dyskinesias (6.2\%).

The total UPDRS score ranged from 8 to 110 (mean=63.2, standard deviation=25.5). Regarding to HY, no patient was in stage 1 , four were in stage $1.5(6.7 \%)$, ten in stage $2(16.7 \%)$, eleven in stage $2.5(18.3 \%)$, twenty-five in stage $3(41.7 \%)$ and ten in stage $4(16.7 \%)$.

The Spearman correlation is presented in Table 2. It is noticed that there are direct correlation between: age $\nu$. duration of disease, age vs. UPDRS total score, age vs. HY stages, educational level vs. Sum, disease duration vs. UPDRS total score, disease duration vs. HY stages, and UPDRS total score vs. HY stages. It is also observed that there was indirect or inversely proportional correlation between: educational level vs. duration of disease, educational level $v$. UPDRS total score, UPDRS total score $v s$. Sum, and between Sum vs. HY stages.

\section{DISCUSSION}

The original study that validated the WOQ-9 questionnaire showed high sensitivity and low specificity to detect WO when compared with the clinical evaluation by neurologists ${ }^{14,19,20}$. The higher the sensitivity the higher is the ability of WOQ-9 to detect WO, which also occurred in our assessed sample. 
Physicians

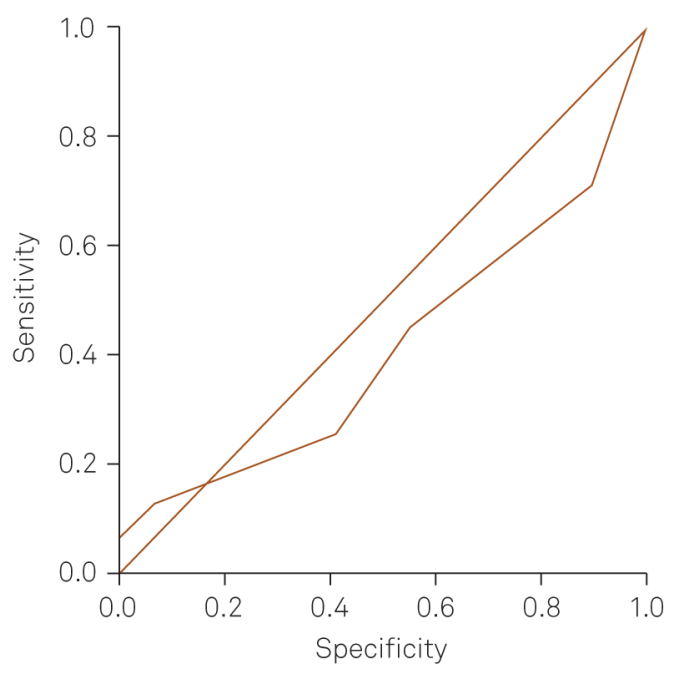

Diagonal segments are produced by ties.
WOQ-9

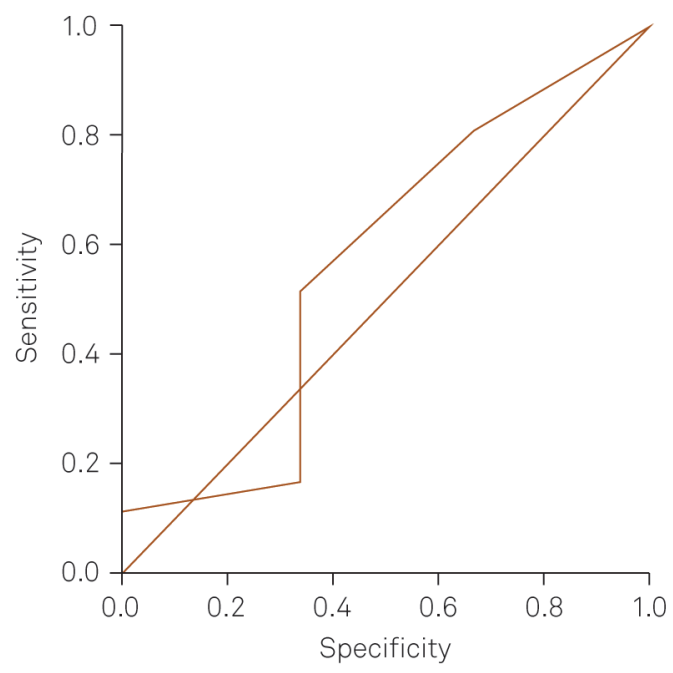

Diagonal segments are produced by ties.

The sensitivity and specificity from the patterned physician's evaluation to detect WO, is demonstrated by the Receiver Operator Curve (ROC) curve on the left (there was no statistical significance). The ROC curve on the right expresses the detection of WO by the WOQ-9 ( $p<0.001$ ).

Figure 1. Sensitivity and specificity of the evaluation by physicians and Nine Items Questionnaire for WO (WOQ-9) for detect wearing-off (WO).

The NPV in our study was high, which implies that the questionnaire is accurate to identify those without WO. On the other hand, the PPV was low indicating that not all WO identified by this instrument actually existed, therefore, it does not qualify the questionnaire as a method to diagnose WO, but rather as one screening method. As a result, this study indicate that our WOQ-9 version is a convenient screening tool to aid physicians to detect WO in PD patients.

In order to increase the identification of motor fluctuations, marked by the predictability and improvement after

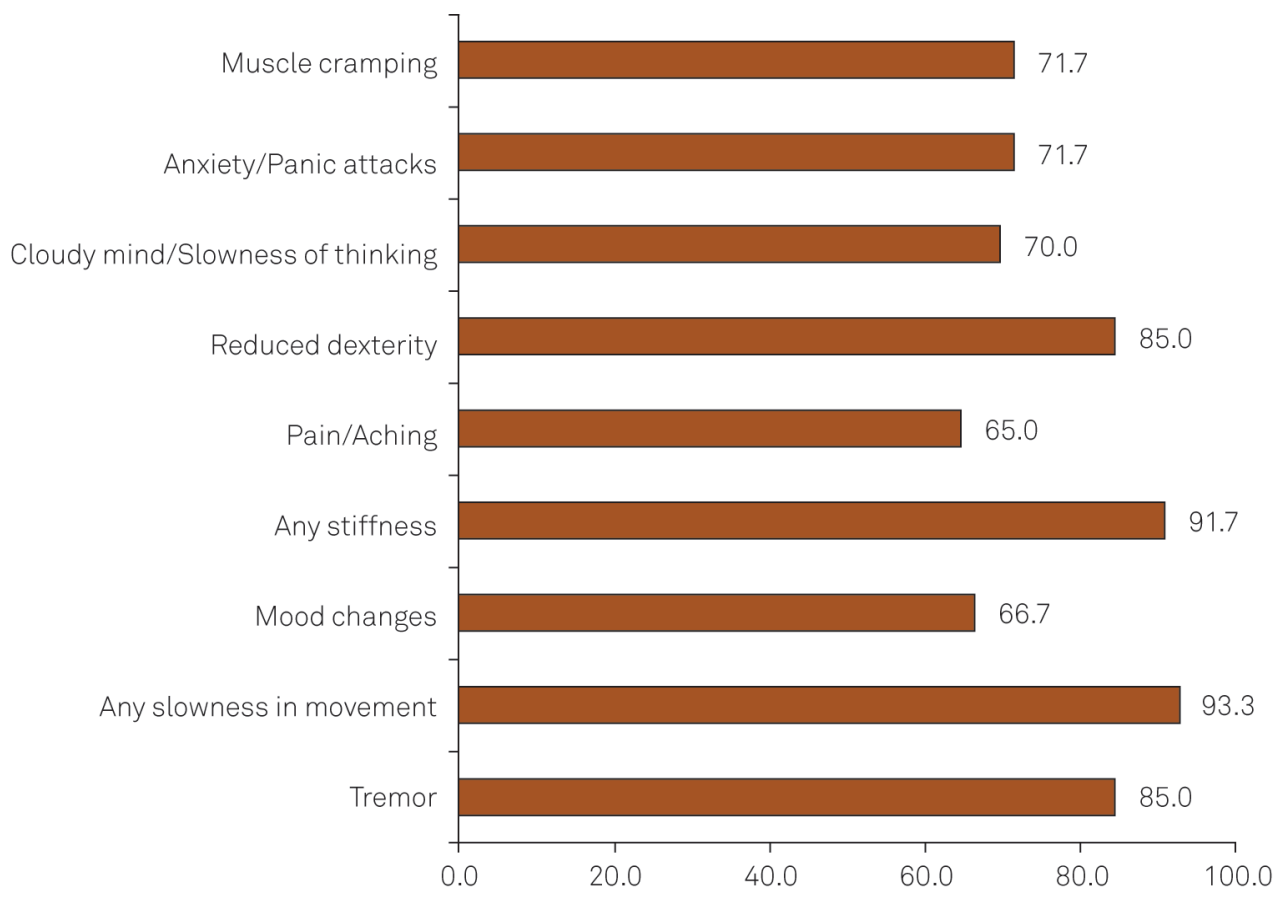

Frequency of the WOQ-9 symptoms reported by patients (values are expressed in percentage).

Figure 2. Frequency of the Nine Items Questionnaire for WO (WOQ-9) symptoms. 


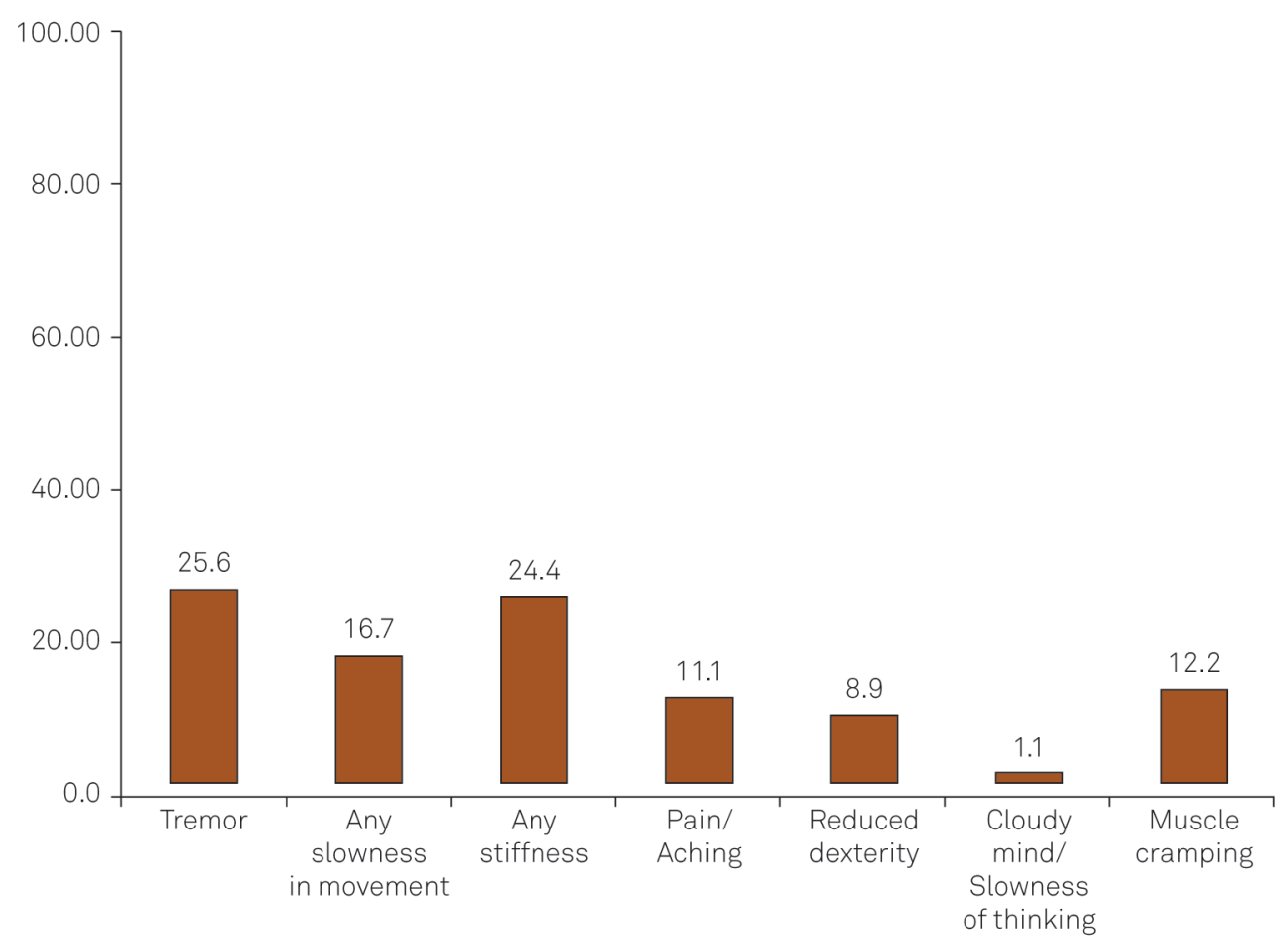

Frequency of the nine symptoms present in the Nine Items Questionnaire for WO (WOQ-9) and pointed out by patients as the most disabling (values are presented in percentage).

Figure 3. The symptoms referred to as the most disabling.

the intake of the next dose of medication, questionnaires including MS and NMS have been developed. The first structured questionnaire to facilitate WO identification was originated from a literature review conducted by ten movement disorders experts ${ }^{11}$.

They met to conceive a prototype patient questionnaire, specifically designed to prove the hypothesis that the sensitivity to detect signs and symptoms of WO by means of evaluation by specialists could be increased using additional methods such as a questionnaire. This questionnaire included 32 symptoms (WOQ-32), seventeen MS and fifteen NMS. In the original study idealized by Stacy and colleagues ${ }^{11}$, WOQ-32 was answered by 300 patients independently, after their routine consultations. They compared the results of the WOQ-32 with those obtained by the standardized clinician assessment and WOQ-32 revealed a sensitivity of $57.1 \%$ to identify WO.
Considering that WOQ-32 was not designed for routinely use, another questionnaire was developed, based on the WOQ-32 and under rigorous statistic methods. Its final composition included nineteen items, $9 \mathrm{MS}$ and $10 \mathrm{NMS}^{12}$. Statistical analysis was made out of the 32 symptoms individually, 13 of which symptoms were selected due to its high positive predictive value, 2 symptoms were selected for its prominence among those cited as disabling. The logarithmic regression analysis of the disabling symptoms added 4 more symptoms, resulting in the WOQ-19. The WOQ-19 has the same sensitivity of the WOQ-32 $2^{12}$.

Finally, a questionnaire with nine symptoms (WOQ-9) was designed to facilitate the patients to answer it, while waiting for their routine consultations ${ }^{13,14}$. The nine items of WOQ-9 were obtained by means of a retrospective statistical analysis of the WOQ-32 symptoms more frequent in the

Table 2. Spearman correlation coefficients analysis.

\begin{tabular}{|c|c|c|c|c|c|c|}
\hline & Age & Educational level & Duration of disease & UPDRS total & Sum & HY \\
\hline Age & 1.000 & & & & & \\
\hline Educational level & $-0.310^{*}$ & 1.000 & & & & \\
\hline Duration of disease & $0.258^{*}$ & $-0.347^{\star \star}$ & 1.000 & & & \\
\hline UPDRS total & $0.466^{* *}$ & $-0.416^{* *}$ & $0.360^{* \star}$ & 1.000 & & \\
\hline Sum & -0.250 & $0.367^{* *}$ & -0.174 & $-0.570^{* *}$ & 1.000 & \\
\hline $\mathrm{HY}$ & $0.423^{* *}$ & -0.224 & $0.275^{*}$ & $0.783^{* *}$ & $-0.492^{* \star}$ & 1.000 \\
\hline
\end{tabular}

${ }^{*} \mathrm{p}<0.05 ;{ }^{*} \mathrm{p}<0.001$. The direct and indirect correlations between data are depicted. The direct correlation was obserced occurred between: age and duration of disease, age and Unified Parkinson's Disease Rating Scale (UPDRS), age and Hoehn and Yahr (HY); educational level and sum; duration of disease and UPDRS, duration of disease and HY and UPDRS and HY. The indirect correlation was observed between: educational level \& duration of disease, educational level and UPDRS; UPDRS and sum and HY and sum. 
patients with WO followed by a canonical discriminant analysis to identify the symptoms that were better predictive to identify WO in the patients with WO and not detected by doctor's assessment from original WOQ-32 study ${ }^{13}$. The sensitivity of WOQ-9 was $96.2 \%$ and specificity $40.9 \%$, proving its usefulness as a screening method ${ }^{14}$. As for the frequency of the WOQ-9 symptoms, either individually or collectively, the NMS were always neglected. This finding was also noticed in other studies, and draws attention to the importance of the presence of the four NMS in WOQ-9, although less referred but of great clinical significance. This is in agreement with the analysis of Stacy et al. ${ }^{14}$, who found that the sensitivity and specificity of WOQ-9 are equivalent to a scale that only uses five motor items. Furthermore, even not listed in the questionnaire, the NMS are always self-reported by the patients, which reflect that these symptoms are substantially uncomfortable for them ${ }^{21}$. On the other hand, the MS are most easily detected and cause a greater impact on PD patients' quality of life. A multicenter study applied a questionnaire of 18 symptoms (9 MS and 9 NMS) in one hundred and sixty patients, of whom $86 \%$ presented WO, and none NMS occurred isolated, but always associated with at least one $\mathrm{MS}^{22}$.

We chose to compare the UPDRS Total score with the WOQ-9 sensitivity, despite of many studies that have compared the UPDRS Question 36 (presence of predictable off periods) with the Wearing-Off Questionnaires sensitivity $^{11,23}$. Recently, a cross-sectional analysis has assessed the clinically important differences between the subscales from UPDRS, as well as the UPDRS motor score and the UPDRS total score. This study revealed better sensitivity of UPDRS total score to detect $\mathrm{NMS}^{24}$.

Finally, the educational level required to answer the questionnaire restricted the sample size. However, enabled that WOQ-9 could be used as it was conceived in its original design. The WOQ-9 was filled out by the patients themselves as they waited for their routine consultations. Another study was conducted in the same institution in order to analyze the applicability of WOQ-19. Although, its methodology allowed achieving a larger sample size, since the questionnaire was read for the patients and the answers registered by the researcher ${ }^{25}$. The average schooling of eight years is similar to other studies conducted in developed countries ${ }^{23,26}$.

In conclusion, the recognition of WO, which may be disabling for some patients, allows its prompt treatment and may delay its progression. Although we used a not validated Brazilian Portuguese translation of WOQ-9, it is a convenient screening tool to aid physicians to detect WO in PD patients, and it is a quick and easy self-administered questionnaire.

\section{References}

1. Olanow CW. Levodopa dopamine replacement strategies in Parkinson's disease: future directions. Mov Disord. 2008;23 Suppl 3:S613-22. http://dx.doi.org/10.1002/mds.22061

2. Stocchi F, Jenner P, Obeso JA. When do levodopa motor fluctuations first appear in Parkinson's disease? Eur Neurol. 2010;63(5):257-66. http://dx.doi.org/10.1159/000300647

3. Parkinson Study Group. Levodopa and the progression of Parkinson's disease. N Engl J Med. 2004;351:2498-508. http://dx.doi.org/ $10.1056 /$ nejmoa033447

4. Obeso JA, Rodriguez-Oroz M, Marin C, Alonso F, Zamarbide I, Lanciego JL et al. The origin of motor fluctuations in Parkinson's disease: importance of dopaminergic innervation and basal ganglia circuits. Neurology. 2004;62(Suppl 1):S17-30. http://dx.doi.org/ 10.1212/wnl.62.1_suppl_1.s17

5. Nutt JG, Holford NH. The response to levodopa in Parkinson's disease: imposing pharmacological law and order. Ann Neurol. 1996;39(5):561-73. http://dx.doi.org/10.1002/ana.410390504

6. Schapira $\mathrm{AH}$, Obeso J. Timing of treatment initiation in Parkinson's disease: a need for reappraisal? Ann Neurol. 2006;59(3):559-62. http://dx.doi.org/10.1002/ana.20789

7. Parkinson Study Group. Entacapone improves motor fluctuations in levodopa-treated Parkinson's disease patients. Ann Neurol. 1997;42(5):747-55. http://dx.doi.org/10.1002/ana.410420511

8. Nissinen H, Kuoppamäki M, Leinonen M, Schapira AH. Early versus delayed initiation of entacapone in levodopa-treated patients with Parkinson's disease: a long-term, retrospective analysis. Eur J Neurol. 2009;16(12):1305-11. http://dx.doi.org/10.1111/j.1468-1331.2009.02726.x

9. Larsen JP, Worm-Petersen J, Siden A, Gordin A, Reinikainen K, Leinonen M. The tolerability and efficacy of entacapone over 3 years in patients with Parkinson's disease. Eur J Neurol. 2003;10(2):137-46. http://dx.doi.org/10.1046/j.1468-1331.2003.00559.x
10. Brooks DJ, Leinonen $M$, Kuoppamäki M, Nissinen $H$. Five-year efficacy and safety of levodopa/ DDCl and entacapone in patients with Parkinson's disease. J Neural Transm. 2008;115(6):843-9. http:// dx.doi.org/10.1007/s00702-008-0025-8

11. Stacy M, Bowron A, Guttman M, Hauser R, Hughes K, Larsen JP et al. Identification of motor and nonmotor wearing-off in Parkinson's disease: comparison of a patient questionnaire versus a clinician assessment. Mov Disord. 2005;20(6):726-33. http://dx.doi.org/ $10.1002 /$ mds. 20383

12. Stacy M, Hauser R. Development of a patient questionnaire to facilitate recognition of motor and non-motor wearing-off in Parkinson's disease. J Neural Transm. 2007;114(2):211-7. http://dx. doi.org/10.1007/s00702-006-0554-y

13. Stacy M, Hauser R, Oertel W, Schapira A, Sethi K, Stocchi F et al. End-of-dose Wearing Off in Parkinson disease: a 9-question survey assessment. Clin Neuropharmacol. 2006;29(6):312-21. http://dx.doi. org/10.1097/01.wnf.0000232277.68501.08

14. Stacy MA, Murphy JM, Greeley DR, Stewart RM, Murck H, Meng X. The sensitivity and specificity of the 9-item Wearing-off Questionnaire. Parkinsonism Relat Disord. 2008;14(3):205-12. http://dx.doi.org/ 10.1016/j.parkreldis.2007.07.013

15. Hughes AJ, Daniel SE, Kilford L, Lees AJ. Accuracy of clinical diagnosis of idiopathic Parkinson's disease: a clinico-pathological study of 100 cases. J Neurol Neurosurg Psychiatry. 1992;55(3):181-4. http://dx.doi.org/10.1136/jnnp.55.3.181

16. Hoehn MM, Yahr MD. Parkinsonism: onset, progression, and mortality. Neurology. 1967;17(5):427-42. http://dx.doi.org/10.1212/ wnl.17.5.427

17. Goetz CG, Tilley BC, Shaftman SR, Stebbins GT, Fahn S, Martinez-Martin $P$ et al. Movement Disorder Society sponsored revision of the Unified Parkinson's Disease Rating Scale (MDS-UPDRS): scale presentation 
and clinimetric testingresults. Mov Disord. 2008;23(15):2129-70. http://dx.doi.org/10.1002/mds.22340

18. Callegari-Jacques SM. Bioestatística: princípios e aplicações. Porto Alegre: Artmed; 2003.

19. Stacy M, Murck H, Meng X. COMPASS-1: A validation study of the 9-question, wearing off questionnaire (WOQ-9). Mov Disord. 2006;21(Suppl):S590.

20. Stacy M. The wearing-off phenomenon and the use of questionnaires to facilitate its recognition in Parkinson's disease. J Neural Transm. 2010;117(7):837-46. http://dx.doi.org/10.1007/s00702-010-0424-5

21. Barbosa ER. Non-motor symptoms in Parkinson's disease. Arq Neuropsiquiatr. 2013;71(4):203-4. http://dx.doi.org/10.1590/0004$282 \times 20130001$

22. Santens P, Noordhout AM. Detection of motor and non-motor symptoms of end-of dose wearing off in Parkinson's disease using a dedicated questionnaire: a Belgian multicenter survey. Acta Neurol Belgica. 2006;106(3):137-41.
23. Martinez-Martin P, Tolosa E, Hernandez B, Badia X. Validation of the "QUICK" questionnaire - a tool for diagnosis of "wearing-off" in patients with Parkinson's disease. Mov Disord. 2008;23(6):830-6. http://dx.doi.org/10.1002/mds.21944

24. Shulman LM, Gruber-Baldini AL, Anderson KE, Fishman PS, Reich SG, Weiner WJ. The clinically important difference on the unified Parkinson's disease rating scale. Arch Neurol. 2010;67(1):64-70. http://dx.doi.org/10.1001/archneurol.2009.295

25. Melo LM, Chien HF, Barbosa ER. Identification of wearing-off manifestations (reduction of levodopa effect) in Parkinson's disease using specific questionnaire and comparison of the results with routine ambulatory evaluations. Arq Neuropsiquiatr. 2010;68(4):506-10. http://dx.doi.org/10.1590/s0004-282×2010000400007

26. Chan A, Cheung YF, Yeung MA, Yeung J, Chung TH, Tsang KL et al. A validation study of the Chinese wearing off questionnaire 9-symptom for Parkinson's disease. Clin Neurol Neurosurg. 2011;11397):538-40. http://dx.doi.org/10.1016/j.clineuro.2011.03.007 